\title{
Convexity of elementary functions with applications to inequalities
}

\begin{abstract}
Milan Jovanović and Vladimir Jovanović
Milan Jovanović received his Ph.D. from the University of Belgrade (Serbia) in 1989. $\mathrm{He}$ is now full professor at the University of Banja Luka (Bosnia and Herzegovina). His main interest is convex optimization.

Vladimir Jovanović received his Ph.D. from the Albert-Ludwigs Universität Freiburg (Germany) in 2004. At present he is assistant professor at the University of Banja Luka (Bosnia and Herzegovina). His research focuses on numerical methods for partial differential equations.
\end{abstract}

\section{Introduction}

Let $I \subset \mathbb{R}$ be an interval. The function $f: I \rightarrow \mathbb{R}$ is convex, if the inequality

$$
f(\lambda x+(1-\lambda) y) \leqslant \lambda f(x)+(1-\lambda) f(y)
$$

holds for all $\lambda \in[0,1]$ and $x, y \in I$. If instead $\leqslant$ in (1) we have $\geqslant$, such a function is concave.

In most textbooks convexity is treated within differential calculus. However, we will see how one can single out convex functions among the basic elementary ones without exploiting differential calculus. This will be achieved by using several inequalities and simple assertions. In addition, some elementary convex functions, as it was pointed out in Jensen's

Konvexität bzw. Konkavität differenzierbarer Funktionen lässt sich bekanntlich leicht mit den Mitteln der Differentialrechnung nachweisen. In der vorliegenden Arbeit wird ein Werkzeugkatalog zusammengestellt, der es erlaubt, Konvexität bzw. Konkavität von Funktionen zu zeigen, die nicht notwendigerweise differenzierbar sind. Beispielsweise genügt es für den Nachweis der Konvexität einer stetigen Funktion $f$ die Gültigkeit der Ungleichung $f((x+y) / 2) \leq(f(x)+f(y)) / 2$ für alle $x, y \in \mathbb{R}$ $\mathrm{zu}$ verifizieren. Mit ihrem Katalog untersuchen die Autoren die Konvexität bekannter elementarer Funktionsklassen in systematischer Weise. Mit Hilfe dieses Formalismus gelingt es ihnen überdies, die Konvexität komplexerer Funktionen relativ leicht einzusehen und daraus bekannte Ungleichungen erneut abzuleiten. 
fundamental paper [5], are not differentiable, such as

$$
x \mapsto \sum_{i=1}^{n}\left|x-x_{i}\right| \quad\left(x_{i} \in \mathbb{R}, \quad i=1, \ldots, n\right)
$$

Moreover, in the same paper Jensen provided an example (attributed to U. Dini and J. Lüroth) of a convex function defined on $(0,1)$, which is not differentiable at any rational point:

$$
f(x)=\sum_{i=1}^{\infty} c_{i}\left|x-x_{i}\right|
$$

Here $c_{i}>0$, the series $\sum_{i=1}^{\infty} c_{i}$ is convergent and $\left\{x_{i}: i \in \mathbb{N}\right\}=\mathbb{Q} \cap(0,1)$. The proof follows from the relation

$$
f_{+}^{\prime}\left(x_{i}\right)-f_{-}^{\prime}\left(x_{i}\right)=2 c_{i},
$$

where $f_{ \pm}^{\prime}$ are the one-sided derivatives of $f$.

It should be mentioned that for any convex function $f$ the derivatives $f_{ \pm}^{\prime}$ exist and $f_{-}^{\prime} \leqslant$ $f_{+}^{\prime}$ in the interior of $I$. Furthermore, $f_{+}^{\prime}=f_{-}^{\prime}$ up to a countable set (see, e.g., [8]).

Besides having the one-sided derivatives, convex functions have another natural property: continuity. More precisely, if $f: I \rightarrow \mathbb{R}$ is convex, then it is continuous in the interior of $I$ (see also [8]).

Instead of convex functions defined by (1), Jensen in [5] considered the functions satisfying (1) only for $\lambda=\frac{1}{2}$ :

$$
f\left(\frac{x+y}{2}\right) \leqslant \frac{f(x)+f(y)}{2} \quad(x, y \in I) .
$$

It turns out that these functions, which are called Jensen-convex (or J-convex) functions, are also convex, provided they are continuous. In the proof of this fact Jensen used the inequality

$$
f\left(\frac{x_{1}+\ldots+x_{n}}{n}\right) \leqslant \frac{f\left(x_{1}\right)+\ldots+f\left(x_{n}\right)}{n}
$$

which today bears his name.

Example 1. The function $f(x)=\frac{1}{x}$ is convex on $(0, \infty)$. Indeed, due to continuity it suffices to prove that $f$ is $J$-convex, namely,

$$
\frac{2}{x+y} \leqslant \frac{\frac{1}{x}+\frac{1}{y}}{2} \quad(x, y>0)
$$

and this is obviously true. 


\section{Operations with convex functions}

We start with an evident assertion.

Theorem 1. If $f, g: I \rightarrow \mathbb{R}$ are convex and $\alpha \geqslant 0$, then $f+g$ and $\alpha f$ are also convex.

The next two simple theorems are stated for the first time in [5].

Theorem 2. Let $f: I \rightarrow J$ and $g: J \rightarrow \mathbb{R}$, where $J \subset \mathbb{R}$ is also an interval. Then:

$$
\begin{aligned}
f \text { convex, g convex and increasing } & \Rightarrow g \circ f \text { convex; } \\
f \text { convex, } g \text { concave and decreasing } & \Rightarrow g \circ f \text { concave; } \\
f \text { concave, } g \text { concave and increasing } & \Rightarrow g \circ f \text { concave; } \\
f \text { concave, } g \text { convex and decreasing } & \Rightarrow g \circ f \text { convex. }
\end{aligned}
$$

Proof. Let us prove only the first statement. For all $x, y \in I$, we have in order

$$
\begin{aligned}
f(\lambda x+(1-\lambda) y) & \leqslant \lambda f(x)+(1-\lambda) f(y), \\
g(f(\lambda x+(1-\lambda) y)) & \leqslant g(\lambda f(x)+(1-\lambda) f(y)) \\
& \leqslant \lambda g(f(x))+(1-\lambda) g(f(y)) .
\end{aligned}
$$

Theorem 3. Let $f: I \rightarrow J, J \subset \mathbb{R}$ with $J=f(I)$. Then:

(i) $f^{-1}$ is convex, if $f$ is convex and decreasing or concave and increasing;

(ii) $f^{-1}$ is concave, if $f$ is convex and increasing or concave and decreasing.

Proof. Suppose that $f$ is convex and increasing. Then $f^{-1}$ is increasing. Consequently, for all $x_{1}, x_{2} \in I$ and $y_{1}=f\left(x_{1}\right), y_{2}=f\left(x_{2}\right)$, it follows that

$$
f^{-1}\left(f\left(\lambda x_{1}+(1-\lambda) x_{2}\right)\right) \leqslant f^{-1}\left(\lambda f\left(x_{1}\right)+(1-\lambda) f\left(x_{2}\right)\right),
$$

that is,

$$
\lambda f^{-1}\left(y_{1}\right)+(1-\lambda) f^{-1}\left(y_{2}\right) \leqslant f^{-1}\left(\lambda y_{1}+(1-\lambda) y_{2}\right) .
$$

One similarly proceeds with the remaining statements.

As an immediate consequence of Theorem 2 and Example 1, we have

Theorem 4. If $f: I \rightarrow(0, \infty)$ is concave, then $\frac{1}{f}$ is convex.

An analogous assertion for a convex function does not hold in general. For example, $f(x)=x^{2}+1$ is convex on $\mathbb{R}$, but $\frac{1}{f}$ is neither convex nor concave. 


\section{Some important inequalities}

In the sequel we will employ the following inequalities:

$$
\begin{array}{rlrl}
\sqrt[n]{a_{1} \ldots a_{n}} & \leqslant \frac{a_{1}+\ldots+a_{n}}{n}, & & a_{i} \geqslant 0 \quad(i=1, \ldots, n) ; \\
a^{\lambda} b^{1-\lambda} & \leqslant \lambda a+(1-\lambda) b, & \lambda \in[0,1], a, b \geqslant 0 ; \\
\left(\frac{a+b}{2}\right)^{\alpha} \leqslant \frac{a^{\alpha}+b^{\alpha}}{2}, & \alpha \geqslant 1, a, b \geqslant 0 .
\end{array}
$$

The first inequality is the celebrated inequality between the arithmetic and geometric mean (AM-GM). More than one hundred proofs are known, and one of them, Cauchy's original proof is included in the beautiful book [1].

The second inequality, often called Young's inequality, is a consequence of (4): assuming first that $\lambda=\frac{m}{n}(m, n \in \mathbb{N}, m \leqslant n)$ is rational, we obtain

or equivalently,

$$
\sqrt[n]{a^{m} b^{n-m}} \leqslant \frac{m a+(n-m) b}{n}
$$

$$
a^{\frac{m}{n}} b^{1-\frac{m}{n}} \leqslant \frac{m}{n} a+\left(1-\frac{m}{n}\right) b .
$$

Passing to the limit in (7), we conclude that (5) holds also for irrational $\lambda \in[0,1]$.

Concerning the proof of the third inequality, note that

$$
a_{1}^{\lambda} b_{1}^{1-\lambda}+a_{2}^{\lambda} b_{2}^{1-\lambda} \leqslant\left(a_{1}+a_{2}\right)^{\lambda}\left(b_{1}+b_{2}\right)^{1-\lambda}
$$

for all positive $a_{i}, b_{i}(i=1,2)$. Indeed, applying inequality (5), we have

$$
\begin{aligned}
& \left(\frac{a_{1}}{a_{1}+a_{2}}\right)^{\lambda}\left(\frac{b_{1}}{b_{1}+b_{2}}\right)^{1-\lambda}+\left(\frac{a_{2}}{a_{1}+a_{2}}\right)^{\lambda}\left(\frac{b_{2}}{b_{1}+b_{2}}\right)^{1-\lambda} \\
& \quad \leqslant \lambda\left(\frac{a_{1}}{a_{1}+a_{2}}\right)+(1-\lambda)\left(\frac{b_{1}}{b_{1}+b_{2}}\right)+\lambda\left(\frac{a_{2}}{a_{1}+a_{2}}\right)+(1-\lambda)\left(\frac{b_{2}}{b_{1}+b_{2}}\right) \\
& \quad=1 .
\end{aligned}
$$

If we set

$$
a_{1}=\frac{1}{2} a^{\alpha}, \quad a_{2}=\frac{1}{2} b^{\alpha}, \quad b_{1}=b_{2}=\frac{1}{2}, \quad \lambda=\frac{1}{\alpha}
$$

in (8), we obtain (6).

In $1888, \mathrm{H}$. Simon published a paper with the proof of an inequality slightly more general than (6). There he made the remark that I.-J. Bienaymé had stated in 1840 similar results, however, without proofs.

\section{Convexity of elementary functions}

Since elementary functions are continuous on their domain of definition, convexity in this case reduces to $J$-convexity. We will see now how one can establish convexity (or concavity) of the most common elementary functions by applying the results from the previous sections. 


\section{Power function}

Let $f(x)=x^{n}$ and $n \in \mathbb{N}$. Then

$$
\left(\frac{x+y}{2}\right)^{n} \leqslant \frac{x^{n}+y^{n}}{2}
$$

holds on $[0, \infty)$ by virtue of $(6)$. Now, it is a simple matter to prove that $f$ is convex on the whole of $\mathbb{R}$ for $n$ even and concave on $(-\infty, 0]$ for $n$ odd.

\section{Root function}

Taking into account that $f(x)=\sqrt[n]{x}$ is the inverse of the power function, Theorem 3 applies.

\section{Exponential function}

Thanks to the AM-GM inequality for $n=2$, we have

$$
e^{\frac{x+y}{2}}=\sqrt{e^{x} e^{y}} \leqslant \frac{e^{x}+e^{y}}{2},
$$

and, consequently, exp is convex on $\mathbb{R}$.

The function $f(x)=a^{x}(a>0)$ is also convex on $\mathbb{R}$ as being a composition of the increasing convex function exp and the convex (linear) function $x \mapsto(\ln a) x$.

\section{Logarithmic function}

Again, since $f(x)=\log _{a} x(a>0, a \neq 1)$ is the inverse of the convex function $x \mapsto a^{x}$, we simply employ Theorem 3 to deduce concavity for $a>1$ and convexity for $0<a<1$.

\section{Generalized power function}

For $f(x)=x^{\alpha}(x>0)$ we distinguish the following cases:

(a) $\alpha \geqslant 1$ :

The corresponding inequality (2) for $f$ is exactly (6).

(b) $0<\alpha<1$ :

In this case $f$ is concave, since $f^{-1}(x)=x^{1 / \alpha}$ is increasing and, according to (a), convex.

(c) $\alpha<0$ :

Utilizing convexity of $x \mapsto \alpha \ln x$, it follows from $f(x)=e^{\alpha \ln x}$ that $f$ is convex. 


\section{Trigonometric functions}

(a) The function $\sin$ is concave on $[0, \pi]$. Indeed, for $x, y \in[0, \pi]$, we have $\sin \frac{x+y}{2} \geqslant 0$. Therefore,

$$
\sin x+\sin y=2 \sin \frac{x+y}{2} \cos \frac{x-y}{2} \leqslant 2 \sin \frac{x+y}{2} .
$$

Hence,

$$
\sin \frac{x+y}{2} \geqslant \frac{\sin x+\sin y}{2} .
$$

Applying $\sin (x+\pi)=-\sin x$, we see that $\sin$ is convex on $[\pi, 2 \pi]$.

(b) From (a) and

$$
\cos x=\sin \left(x+\frac{\pi}{2}\right)
$$

we conclude that cos is convex on $\left[\frac{\pi}{2}, \frac{3 \pi}{2}\right]$, and concave on $\left[0, \frac{\pi}{2}\right]$ and $\left[\frac{3 \pi}{2}, 2 \pi\right]$.

(c) $\tan$ is convex on $\left[0, \frac{\pi}{2}\right)$. To see this, we utilize concavity of $\cos$ on $\left[0, \frac{\pi}{2}\right)$ and the AM-GM inequality to obtain

$$
\sqrt{\cos x \cos y} \leqslant \frac{\cos x+\cos y}{2} \leqslant \cos \frac{x+y}{2} .
$$

Finally, for $x, y \in\left[0, \frac{\pi}{2}\right)$, it follows that

$$
\tan \left(\frac{x+y}{2}\right)=\frac{\sin \frac{x+y}{2}}{\cos \frac{x+y}{2}}=\frac{2 \sin \frac{x+y}{2} \cos \frac{x+y}{2}}{2 \cos ^{2} \frac{x+y}{2}} \leqslant \frac{\sin (x+y)}{2 \cos x \cos y}=\frac{\tan x+\tan y}{2} .
$$

As an odd function, tan is concave on $\left(-\frac{\pi}{2}, 0\right]$.

\section{Inverse trigonometric functions}

Here the application of Theorem 3 and previous results allow us to deduce intervals of convexity and concavity for the functions arcsin, arccos, arctan.

\section{Hyperbolic functions}

(a) The function sinh is convex on $[0,+\infty)$. This follows from

$$
\sinh x+\sinh y=2 \sinh \left(\frac{x+y}{2}\right) \cosh \left(\frac{x-y}{2}\right) \geqslant 2 \sinh \left(\frac{x+y}{2}\right) .
$$

sinh is concave on $(-\infty, 0]$, since it is an odd function.

(b) The function cosh is convex on $\mathbb{R}$ being a sum of two convex functions. Note that $\ln$ cosh is also convex on $\mathbb{R}$ by virtue of Theorem 2 . Inequality (2) for this function implies that

$$
\cosh \left(\frac{x+y}{2}\right) \leqslant \sqrt{\cosh x \cosh y} .
$$


(c) From (9) one infers that for all $x, y \geqslant 0$,

$$
\tanh \left(\frac{x+y}{2}\right)=\frac{2 \sinh \frac{x+y}{2} \cosh \frac{x+y}{2}}{2 \cosh ^{2} \frac{x+y}{2}} \geqslant \frac{\sinh (x+y)}{2 \cosh x \cosh y}=\frac{\tanh x+\tanh y}{2},
$$

which means that tanh is concave on $[0, \infty)$. Convexity on $(-\infty, 0]$ follows from the fact that it is an odd function.

\section{Applications to inequalities}

\subsection{Henrici's and Ky Fan's inequalities}

Two interesting inequalities can be derived by using concavity of tanh on $[0, \infty)$. First, note that the function $f(x)=\frac{1}{1+e^{x}}$ is convex on $[0, \infty)$, due to

$$
f(x)=\frac{1}{2}-\frac{1}{2} \tanh \frac{x}{2}
$$

Jensen's inequality (3) for $f$ reduces to the inequality of P. Henrici [4]

$$
\frac{n}{1+\sqrt[n]{x_{1} \ldots x_{n}}} \leqslant \sum_{i=1}^{n} \frac{1}{1+x_{i}} \quad\left(x_{i} \geqslant 1, \quad i=1, \ldots, n\right)
$$

Note further that $f$ is decreasing. Therefore,

$$
f^{-1}(x)=\ln \frac{1-x}{x}
$$

is convex on $\left(0, \frac{1}{2}\right)$. Jensen's inequality (3) applied to $f^{-1}$ leads to the inequality of Ky Fan

$$
\frac{\prod_{i=1}^{n} x_{i}}{\left(\sum_{i=1}^{n} x_{i}\right)^{n}} \leqslant \frac{\prod_{i=1}^{n}\left(1-x_{i}\right)}{\left(\sum_{i=1}^{n}\left(1-x_{i}\right)\right)^{n}}, \quad 0<x_{i} \leqslant \frac{1}{2} \quad(i=1, \ldots, n) .
$$

This shows how closely related the inequalities of Henrici and Ky Fan are.

Interestingly, Henrici's inequality was posed as problem No. 245 in Elemente der Mathematik in 1955. On the other hand, Ky Fan's inequality has originally been stated without proof in [2] as an unpublished result of Ky Fan, accompanied with a hint of using mathematical induction.

\subsection{Several geometric inequalities}

Let us denote by $P$ the area of a triangle with the side lengths $a, b, c$, the angles $\alpha, \beta, \gamma$, and the height lengths $h_{a}, h_{b}, h_{c}$.

Thanks to concavity and positivity of the function $\sin$ on $(0, \pi)$, we conclude by virtue of Theorem 4 that the function $1 / \sin$ is convex on the same interval. Jensen's inequality (3) with $n=3$ in this case yields

$$
\frac{1}{\sin \frac{\alpha+\beta+\gamma}{3}} \leqslant \frac{\frac{1}{\sin \alpha}+\frac{1}{\sin \beta}+\frac{1}{\sin \gamma}}{3}
$$


Therefore,

$$
\frac{1}{\sin \alpha}+\frac{1}{\sin \beta}+\frac{1}{\sin \gamma} \geqslant 2 \sqrt{3}
$$

Now, from

$$
a^{2}+b^{2}+c^{2} \geqslant a b+b c+c a=2 P\left(\frac{1}{\sin \alpha}+\frac{1}{\sin \beta}+\frac{1}{\sin \gamma}\right),
$$

we get Weitzenböck's inequality [9]

$$
4 \sqrt{3} P \leqslant a^{2}+b^{2}+c^{2} .
$$

Note that from

$$
6 P=a h_{a}+b h_{b}+c h_{c} \leqslant a b+b c+c a \leqslant a^{2}+b^{2}+c^{2}
$$

follows

$$
4 \sqrt{3} P \leqslant \frac{2}{\sqrt{3}}\left(a^{2}+b^{2}+c^{2}\right) .
$$

Consequently, Weitzenböck's inequality is an improvement of the trivial inequality (10). Many estimates for $4 \sqrt{3} P$ are known. For example, Pólya and Szegö proved in [6] that

$$
4 \sqrt{3} P \leqslant 3 \sqrt[3]{a^{2} b^{2} c^{2}}
$$

Having in mind that

$$
3 \sqrt[3]{a^{2} b^{2} c^{2}} \leqslant a^{2}+b^{2}+c^{2}
$$

we see that the estimate (11) is sharper than Weitzenböck's inequality. Here we show a proof of (11), which partially employs ideas of H. Flanders [3].

The function $f=\ln \sin$ is a composition of two concave functions with $\ln$ additionally being increasing. Thus, $f$ is concave. Jensen's inequality applied to $-f$ implies

$$
\sin \alpha \sin \beta \sin \gamma \leqslant \frac{3}{8} \sqrt{3}
$$

and (11) easily follows from the evident identity

$$
P^{3}=\frac{1}{8}(a b c)^{2} \sin \alpha \sin \beta \sin \gamma .
$$

We leave as an exercise to prove the inequality (see [7]):

$$
\sin \frac{\alpha}{2} \sin \frac{\beta}{2} \sin \frac{\gamma}{2} \leqslant \frac{1}{8}
$$




\section{References}

[1] Aigner, M.; Ziegler, G.M.: Proof from THE BOOK. Springer-Verlag, 2003.

[2] Beckenbach, E.F.; Bellman, R.: Inequalities. Springer-Verlag, Berlin, 1961.

[3] Flanders, H.: Review: Problems and theorems in analysis. Bull. Amer. Math. Soc. 84 (1978), 53-62.

[4] Neue Aufgaben. Elem. Math. 10 (1955), 92.

[5] Jensen, J.L.W.V.: Sur les fonctions convexes et les inégalités entre les valeurs moyennes. Acta Math. 30 (1906), 175-193.

[6] Pólya, G.; Szegö, G.: Problems and theorems in analysis. Vol. I. Springer-Verlag, 1972.

[7] Radó, T.: On mathematical life in Hungary. Amer. Math. Monthly 37 (1932), 85-90.

[8] Roberts, A.W.; Varberg, D.: Convex Functions. Academic Press, 1973.

[9] Weitzenböck, R.: Über eine Ungleichung in der Dreiecksgeometrie. Math. Z. 5 (1919), 137-146.

Milan Jovanović and Vladimir Jovanović

Faculty of Sciences

University of Banja Luka

Mladena Stojanovića 2

51000 Banja Luka, Bosnia and Herzegovina

e-mail: jaglikaj@yahoo.com

e-mail: vlajov@blic.net 